\title{
Deep learning for semi-automated unidirectional measurement of lung tumor size in $\mathrm{CT}$
}

MinJae Woo ${ }^{1}$, A. Michael Devane ${ }^{2}$, Steven C. Lowe², Ervin L Lowther ${ }^{2}$ and Ronald W. Gimbel ${ }^{1 *}$

\begin{abstract}
Background: Performing Response Evaluation Criteria in Solid Tumor (RECISTS) measurement is a non-trivial task requiring much expertise and time. A deep learning-based algorithm has the potential to assist with rapid and consistent lesion measurement.
\end{abstract}

Purpose: The aim of this study is to develop and evaluate deep learning (DL) algorithm for semi-automated unidirectional CT measurement of lung lesions.

Methods: This retrospective study included 1617 lung CT images from 8 publicly open datasets. A convolutional neural network was trained using 1373 training and validation images annotated by two radiologists. Performance of the $\mathrm{DL}$ algorithm was evaluated 244 test images annotated by one radiologist. DL algorithm's measurement consistency with human radiologist was evaluated using Intraclass Correlation Coefficient (ICC) and Bland-Altman plotting. Bonferroni's method was used to analyze difference in their diagnostic behavior, attributed by tumor characteristics. Statistical significance was set at $p<0.05$.

Results: The DL algorithm yielded ICC score of 0.959 with human radiologist. Bland-Altman plotting suggested 240 (98.4\%) measurements realized within the upper and lower limits of agreement (LOA). Some measurements outside the LOA revealed difference in clinical reasoning between DL algorithm and human radiologist. Overall, the algorithm marginally overestimated the size of lesion by $2.97 \%$ compared to human radiologists. Further investigation indicated tumor characteristics may be associated with the DL algorithm's diagnostic behavior of over or underestimating the lesion size compared to human radiologist.

Conclusions: The DL algorithm for unidirectional measurement of lung tumor size demonstrated excellent agreement with human radiologist.

Keywords: Lung Cancer, Response Evaluation Criteria in Solid Tumors 1.1, Semi-automated annotation, Tumor Measurement, Deep learning

\footnotetext{
* Correspondence: rgimbel@clemson.edu

'Department of Public Health Sciences, Clemson University, 501 Edwards Hall, Clemson, SC 29634, USA

Full list of author information is available at the end of the article
}

(c) The Author(s). 2021 Open Access This article is licensed under a Creative Commons Attribution 4.0 International License, which permits use, sharing, adaptation, distribution and reproduction in any medium or format, as long as you give appropriate credit to the original author(s) and the source, provide a link to the Creative Commons licence, and indicate if changes were made. The images or other third party material in this article are included in the article's Creative Commons licence, unless indicated otherwise in a credit line to the material. If material is not included in the article's Creative Commons licence and your intended use is not permitted by statutory regulation or exceeds the permitted use, you will need to obtain permission directly from the copyright holder. To view a copy of this licence, visit http://creativecommons.org/licenses/by/4.0/ The Creative Commons Public Domain Dedication waiver (http://creativecommons.org/publicdomain/zero/1.0/) applies to the data made available in this article, unless otherwise stated in a credit line to the data. 


\section{Background}

Response evaluation of cancer therapeutics is often a prerequisite to various clinical decisions in cancer treatment. Response Evaluation Criteria in Solid Tumor 1.1 (RECIST 1.1) is the predominant clinical guideline to determine whether tumors in cancer patients responded to treatment, stay the same, or worsened during cancer therapeutics [1-4]. Application of RECIST guideline involves a series of tumor size measurements, which is an important surrogate marker of therapeutic efficacy. Consistent and accurate measurements of tumor size are essential with their direct impact on cancer treatment management.

Performing RECISTS measurement is a non-trivial task requiring a great deal of expertise and time by a highly trained radiologist. Multiple reports have indicated that the tumor size measurements using computed tomography (CT) scans are subjected to intra- and interobserver variability with various environmental factors causing the variability [5-12]. To address these challenges, researchers have attempted to develop systems to assist with consistent lesion measurement through automated lesion segmentation or masking for CT images [13-18]. Most studies used segmentation techniques with probabilistic approaches to drawing lesion boundaries. However, segmentation results are often noncomparable to radiologist measurements as radiologists use unidirectional measurement. Conversion of segmentation results into unidirectional measurement poses challenges as the task requires additional clinical reasoning to decide the start point, end point, and longest axis of the measurement, Fig. 1. Performing segmentation often takes longer than performing unidirectional measurement by human radiologists; this incurs additional costs on the acquisition of training data for any automated system for measurement.

In this study, we propose a new approach for application of a deep learning (DL) algorithm on semiautomated CT measurement of lung lesions. To the best of our knowledge, this study was the first to propose semi-automated measurement of tumor without involving segmentation or masking process. The purpose is to develop a tool performing measurement comparable to radiologist measurement, which has a potential to assist radiologists with consistent RECIST annotation by improving inter-observer measurement variability. We also investigated how different lesion types challenge the proposed application of the DL algorithm.

\section{Methods}

\section{Image data sets}

We reviewed 8 publicly open datasets with 146,403 cross-sectional lung CT images from various institutions (Table 1) [19-27]. A total of 1,617 cross-sectional lung
CT images were included in this study after applying the following inclusion criteria: (a) selected lesion should be measurable under RECIST 1.1 (b) selected image file contains complete Digital Imaging and Communications in Medicine (DICOM) pixel data with no corruption (c) lesion size should differ by $20 \%$ when compared to the previously selected images if selected from the same patient (d) selected image has at least $5 \mathrm{~mm}$ spacing to the previously selected images if selected from the same patient. Additionally, DICOM metadata relevant to image processing (e.g. pixel spacing, window/level settings) was inspected for all selected image files. In the selected images, CT scanning parameters were as follows: tube voltage of 100, 120, 130, and $140 \mathrm{kV}$, and tube current $30-543 \mathrm{~mA}$, and slice thickness of 1.0-6.0 mm.

\section{Reference RECIST measurements}

Three experienced board-certified radiologists who regularly perform treatment response evaluation participated in training and evaluation of the proposed DL algorithm, Fig. 2. Radiologist 1 (MD) selected CT images for the study according to the eligibility criteria. The 1,617 CT images selected by the Radiologist 1 were randomly assigned to training set, validation set, and test set through dataset-wise block randomization, Table 1 . The following ratio was used as suggested in the potentially relevant studies using deep learning: $70 \%$ training, $15 \%$ validation, $15 \%$ test [28-30]. Radiologist 2 (SL) and Radiologist 3 (EL) performed measurement on images from the training and validation sets, resulting in the exclusion of additional 18 images due to inter-observer variability regarding the RECIST measurability on the images initially selected by Radiologist 1 . As a result, the training dataset included images deemed measurable by all three radiologists. The test set was labeled by Radiologist 1. For clarity, there was no overlap between training and test data. The radiologist who labeled the test dataset was ruled out during the training process. The participating radiologists did not have access to measurements performed by other radiologists to prevent observer bias. The reference measurements were performed between October 2018 to June 2019.

\section{Semi-automated measurement using deep learning algorithm}

The DL network for automatic lesion measurement consisted of three consecutive convolutional neural networks that labeled whether the size of a target lesion in a given image frame was larger or smaller than 32 pixels. We assumed that, if the DL network failed to classify, the failure occurred because the lesion size approximated to 32 pixels. 

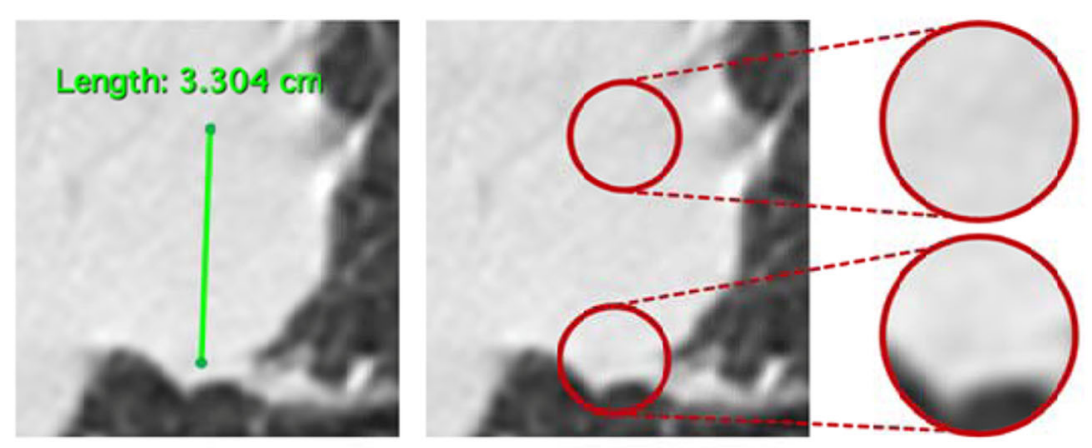

(a)
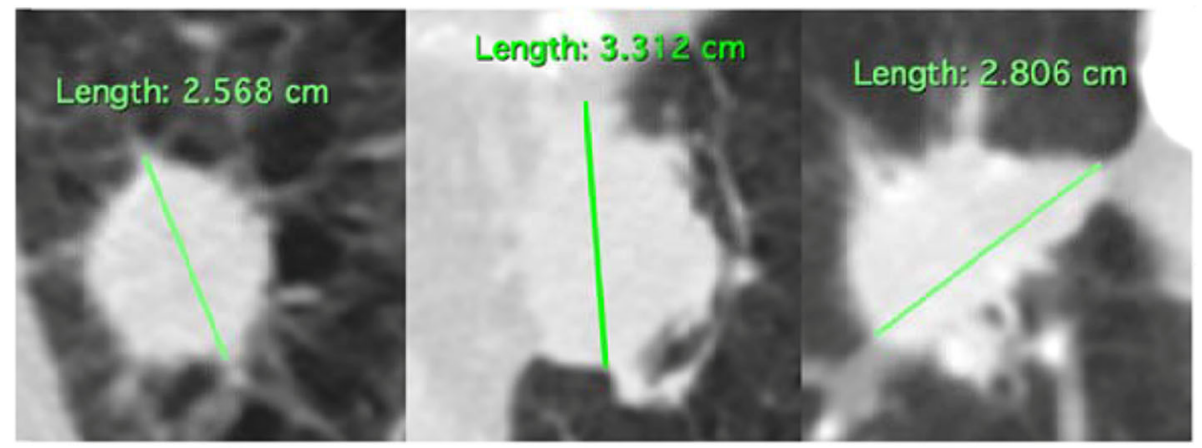

(b)

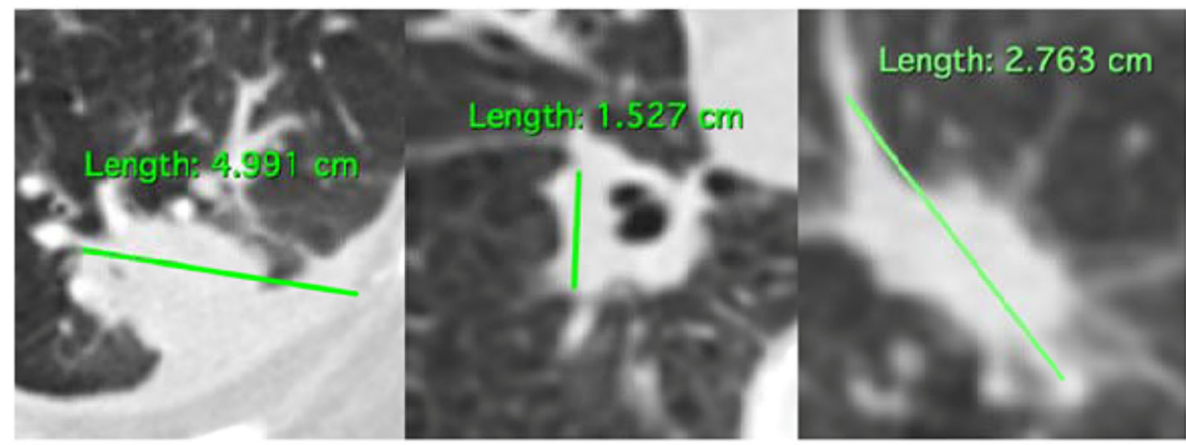

(c)

Fig. 1 Challenges associated with lesion segmentation and its conversion to unidirectional RECIST measurement. a Automated lesion measurement is challenged by the absence of visual distinction between beginning and ending points and their surrounding areas. $\mathbf{b}$ Lesion boundaries are visually distinct and well-defined; both segmentation and conversion of segmentation into measurement can easily be automated using existing techniques and tools. c Both segmentation and conversion of segmentation into measurement require a significant amount of clinical reasoning, which poses challenges to the idea of automated measurement through segmentation

The training data preparation was performed by resizing each CT image so that its target lesion would have a size of 32 pixels. The unidirectional measurements in centimeters were converted into measurements in pixels $\left(M_{p x}\right)$. The images were then magnified by $32 / M_{p x}$ times using bicubic interpolation as a differentiable sampler for the different magnifications [31, 32]. Each target lesion was cropped in a 128-by-128 pixel frame using the center point of measurement as a frame center. The training dataset was generated through image augmentation techniques including zooming in/out, horizontal/vertical shifting of the target lesion in an image frame. Using the various magnifications, the DL network was trained to predict whether a lesion in a 128-by-128 pixel frame is larger or smaller than 32 pixels. The augmentation was also intended to improve the resilience of classification by training the DL algorithm with target lesions off the center of the image frame [33]. The convolutional neural network 


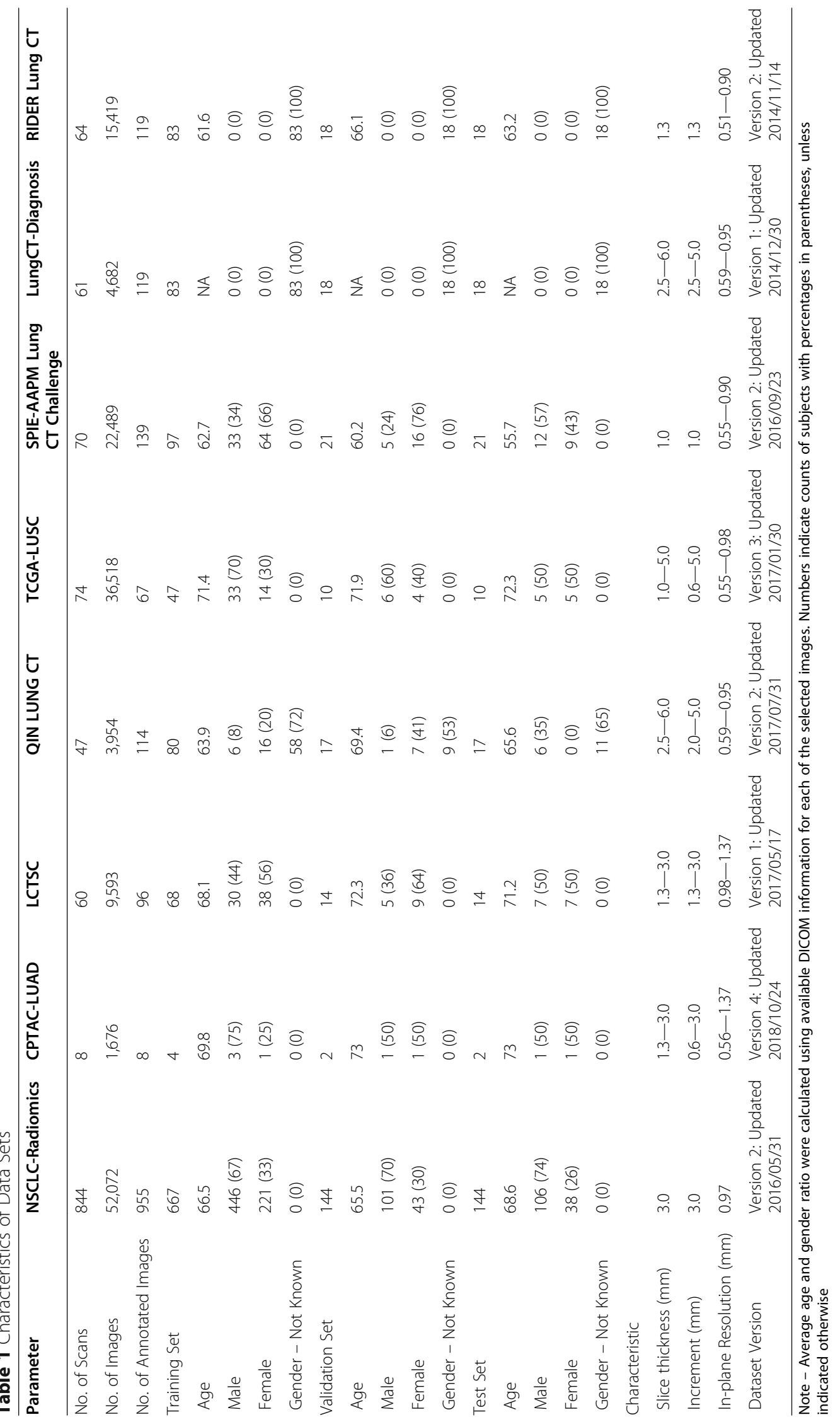




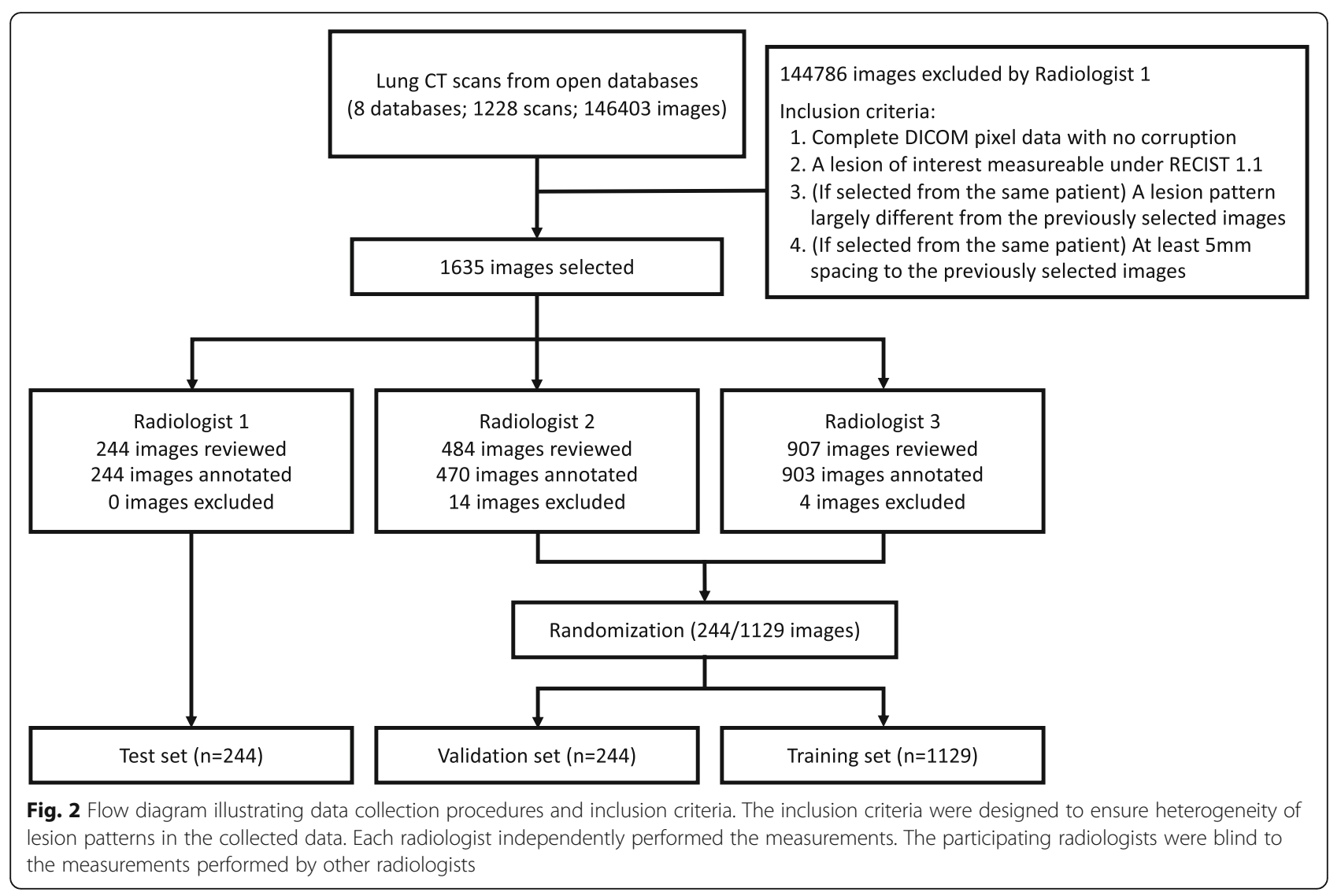

was trained for 500 iterations with a batch size of 32; the model with the highest validation accuracy was selected. The training process was not stratified by the lesion characteristics. A single DL classifier was utilized for both training and inference throughout the study.

The proposed method was semi-automatic; the algorithm was first given with an arbitrary point within a target lesion to perform the RECIST measurements. Once the arbitrary point was acquired, the algorithm utilized the point as a frame center to cropped 128-by-128 pixel image frames containing target lesion with various magnifications, Fig. 3. Upon identification of magnification where the classification failed, a numerical value of measurement was calculated using the magnification and DICOM pixel spacing tag. The codes are available at https:/github.com/ minjaewoo/Semiautomated-CT-Measurement.

\section{Statistical analysis}

Reliability of measurements by the DL algorithm was assessed with the intraclass correlation coefficient (ICC) between automatically and manually obtained measurements for images from the test set. The ICC was calculated using a two-way random-effects model that characterized absolute agreement to account for both lesion-wise effect (target effect) and radiologist-wise effect (rater effect) for evaluation comparable to the previous studies on inter-observer variability in $\mathrm{CT}$ measurement [11, 34].

Bland-Altman plotting with $95 \%$ limits of agreement was produced by averaging lesion size between the human reader and DL algorithm to demonstrate the agreement between the measurements produced by the human reader and DL algorithm [35]. The percentage differences in measurement between the human and algorithm were visualized in a histogram, Fig. 4.

Additional statistical analyses were performed to identify the effect of lesion invasion type on variability between the human radiologist and the DL algorithm. Bonferroni's method was used for pairwise comparison of measurement difference by type of tumor invasion. Statistical significance was set at $p<0.05$.

\section{Results}

\section{Characteristic of data sets}

Mean ages of patients in the training, validation, and test sets were 66.9, 66.1, and 67.1, respectively, Table 1 . Gender information extracted from DICOM metadata suggested more male $(62 \%)$ than female (38\%) representation in selected CT images. Average lesion sizes annotated by the human radiologists for training, validation, and test sets were $3.08 \mathrm{~cm}, 3.26 \mathrm{~cm}$, and $2.99 \mathrm{~cm}$, respectively, Table 2. As intended, Radiologist 1 annotated 244 

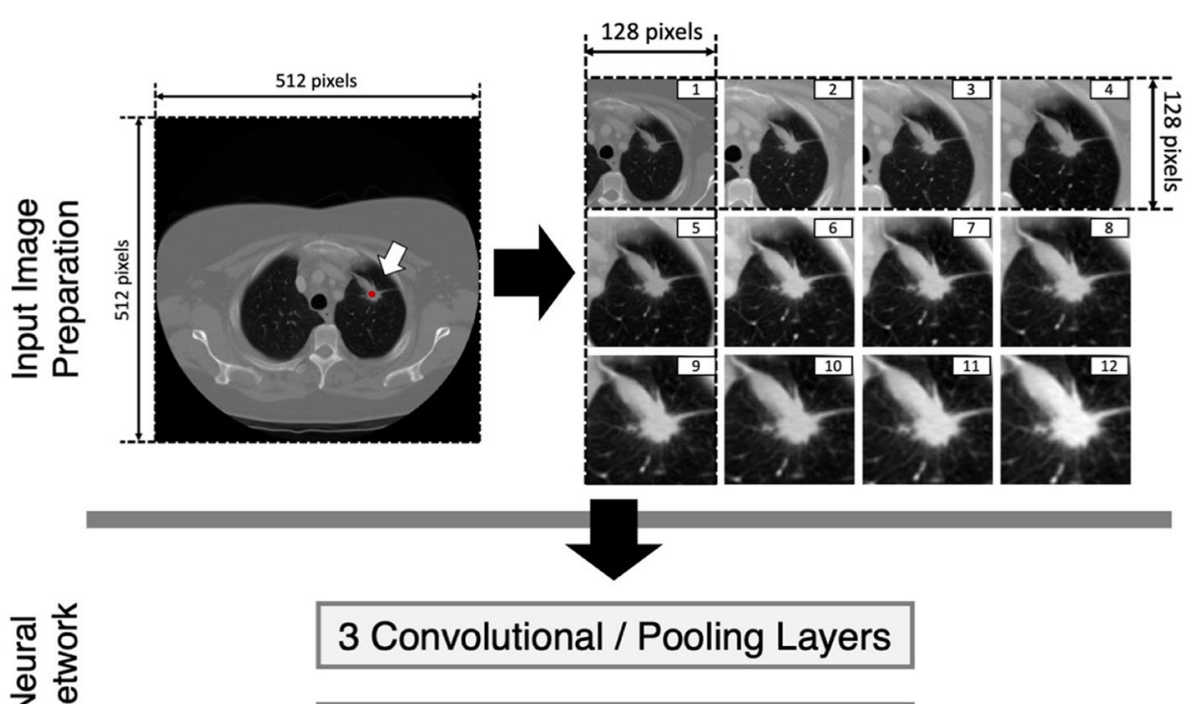

\section{Fully Connected Layer / Softmax}

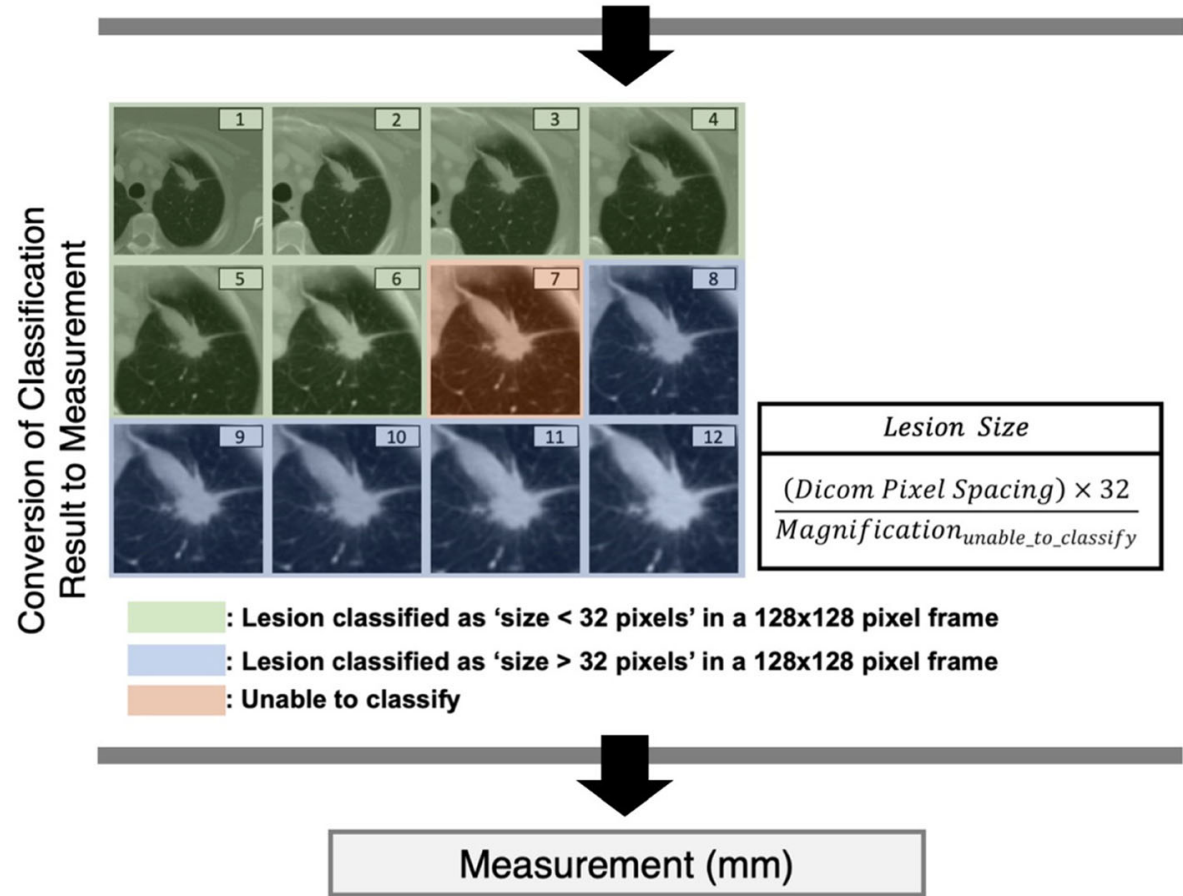

Fig. 3 Overview of deep learning algorithm to perform unidirectional lesion measurement. In the shown example, the input image was augmented into 16 images at various magnifications, using the arbitrary input point (stained in red, pointed by arrow) in the target lesion. The neural network classified each augmented image whether the containing lesion size is larger or smaller than 32 pixels within a 128-by-128 pixel frame. Upon identification of a magnification inducing classification failure, the magnification and pixel spacing information were used to determine the final measurement

images from the test set. Radiologist 2 and Radiologist 3 performed measurements on training and validation sets, which resulted in 903 and 470 images annotated by Radiologist 2 and Radiologist 3, respectively. The difference in the number of images annotated by each radiologist was mainly attributed to different measurement pace between the radiologists. The proposed data augmentation resulted in a total of 142,254 images for training. The augmented training data included 71,127 images with lesion size smaller than 32 pixels and 71,127 images with lesion size larger than 32 pixels within a 128-by-128 pixel frame.

\section{DL algorithm performance}

The DL algorithm achieved an ICC score of $0.959(95 \%$ CI: $0.947,0.967)$ with Radiologist 1 when performing measurements on the same set of 244 CT images, 


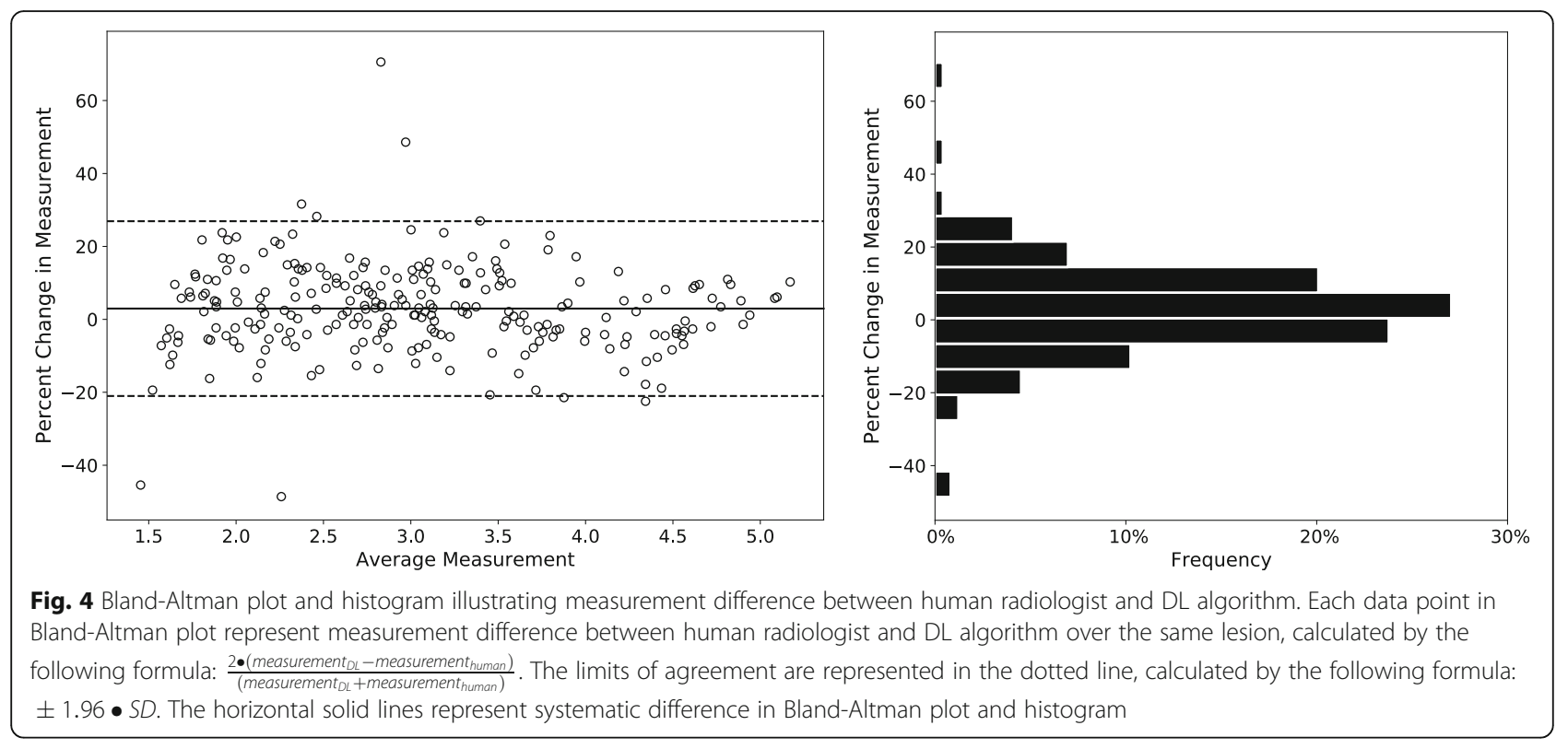

Table 2. Bland-Altman plotting revealed a mean percent difference (systematic difference) of $2.97 \%$ between human and DL algorithm; overall, the algorithm marginally overestimated the size of lesion by $2.97 \%$ compared to human radiologists. Bland-Altman upper and lower limits of agreement (LOA) were realized at 24.3 and $20.7 \%$. The plot also revealed a total of 6 measurements outside the lower and upper LOA. Although previous studies reported that the percent differences marginally outside the LOA are not unusual among human observers [12], some percent differences were unusually high and possibly indicated the algorithm failure. We have identified 4 lesions that caused unusually high measurement difference between the DL algorithm and human radiologist; there were two lesions above upper LOA with 48.5 and $70.6 \%$ measurement difference, and two lesions below lower LOA with 45.4 and $48.6 \%$ measurement difference. The lesions that caused the outlier measurement difference between human and DL algorithm were presented and compared in Fig. 5. For technical details on how the start point, end point, and longest axis were determined for the presented measurements by DL algorithm, see Supplemental Material 1.

The Bland-Altman analysis indicated no heteroscedasticity issue; the visualization suggested no evidence of increasing measurement difference between human and DL algorithm with an increase in average measurement. A benchmark to test the performance of the DL algorithm indicated that performing a single measurement by the algorithm takes on average of $2.2 \mathrm{~s}$ per lesion when tested on NVIDIA Jetson TX2 platform, whereas the participating radiologists spent on average of $17.8 \mathrm{~s}$ per lesion.

\section{Effect of invasion type on performance}

Bland-Altman analysis suggested different diagnostic behavior between the DL algorithm and human radiologist when performing measurements. Overall, the algorithm tended to overestimate the size of lesion by $2.97 \%$ compared to the human radiologist. Further comparisons stratified by tumor characteristics indicated that invasion type may be associated with diagnostic behavior of the

Table 2 Reader statistics and inter-observer variability between human radiologist and DL algorithm

\begin{tabular}{llll}
\hline Reader & Image Use & Number of Annotated Images & Average Measurement (cm) \\
\hline Radiologist 1 & Test & 244 & $2.99 \pm 0.93(1.57-4.91)$ \\
Radiologist 2 & Training & 734 & $3.17 \pm 0.96(1.51-5.00)$ \\
& Validation & 159 & $3.21 \pm 0.98(1.50-4.99)$ \\
Radiologist 3 & Training & 395 & $2.92 \pm 0.88(1.49-4.94)$ \\
& Validation & 85 & $3.35 \pm 0.89(1.56-4.79)$ \\
DL Algorithm & Test & 244 & $3.07 \pm 0.91(1.37-5.44)$ \\
Radiologist 1 \& DL Algorithm ICC: 0.959 (95\% Cl: 0.947, 0.967) & &
\end{tabular}

Note - Average Measurement \pm Standard Deviation. Numbers in parentheses represent range consisting of (minimum observed value - maximum observed value). ICC denotes intraclass correlation coefficient. The ICC score is based on a two-way random-effects model. Cl denotes confidence interval. 


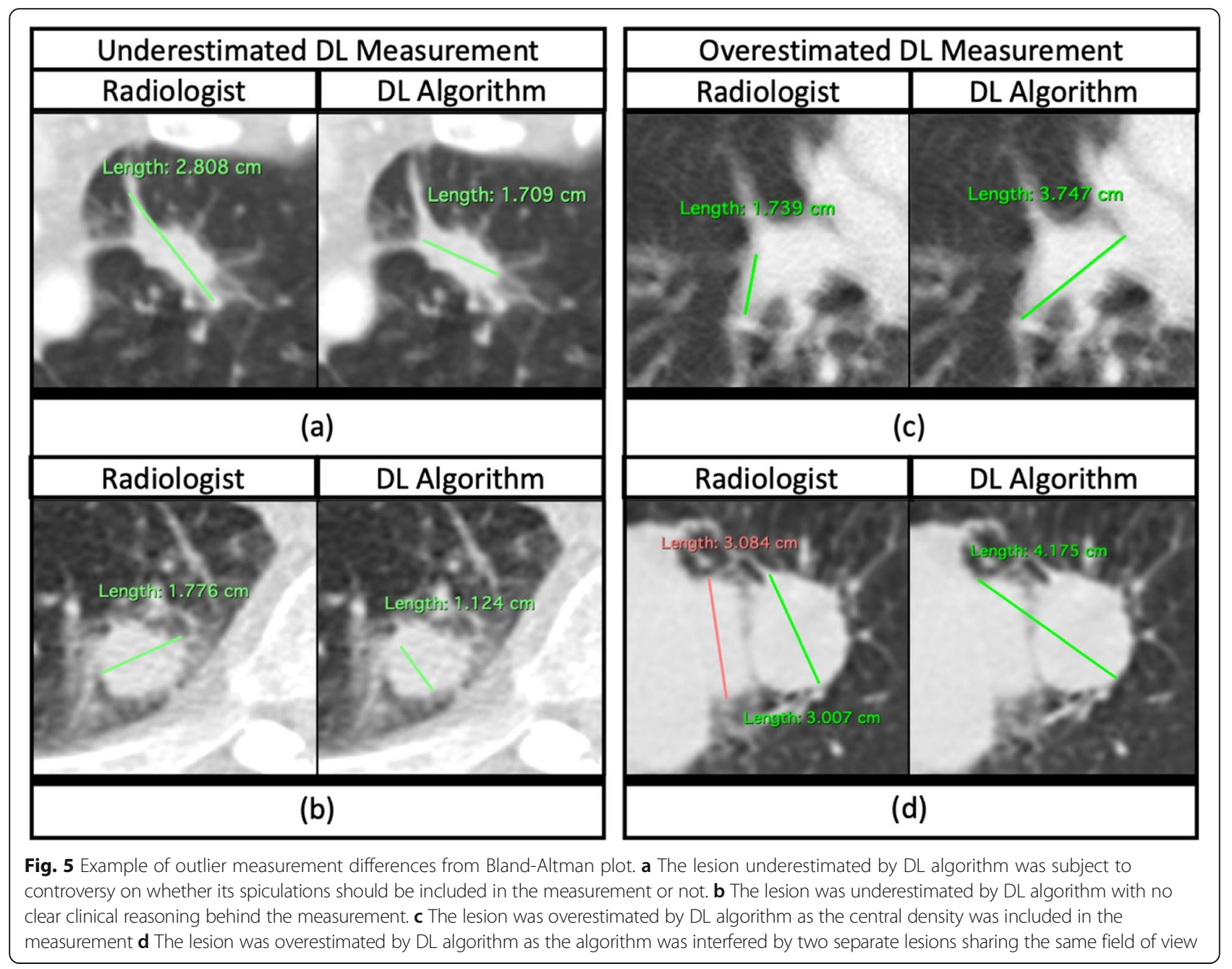

DL algorithm resulting in overestimation or underestimation of lesion size. Specifically, a lesion-wise effect on the difference in diagnostic behavior between the human radiologist and DL algorithm was identified for the following invasion type classification: (A) Parietal pleura/ chest wall invasion (B) Mediastinal pleural invasion (C) Endobronchial invasion less than $2 \mathrm{~cm}$ distal to the carina (D) Invasion associated collapse (atelectasis) (E) Peripheral invasion surrounded by lung (F) Diaphragm invasion, Fig. 6 [36]. The Bonferroni pairwise comparison suggested that the measurements by the algorithm are more likely to be overestimated compared to human radiologist when measuring (B) tumor invading mediastinal pleural and (D) tumor associated collapse (atelectasis) or obstructive pneumonia, as compared to when measuring $(\mathrm{C})$ endobronchial tumor less than $2 \mathrm{~cm}$ distal to the carina and (F) tumor invading diaphragm.

\section{Discussion}

This was the first study to perform semi-automated measurements without masking or segmentation process. The proposed algorithm facilitated the use of unidirectional measurement throughout its training process, which significantly reduced the cost of data acquisition. It yielded output comparable to a human radiologist's standard RECIST measurement used in daily clinical practice. The proposed methodology has the potential to assist other anatomic measurements in the images with metadata containing information on pixel spacing.

The inter-observer agreement rate between the DL algorithm and human radiologist was 0.959 when evaluated using ICC. Its performance is consistent with the previously published study by McErlean in which 17 radiologists measured the same 320 lesions to evaluate inter-observer variability and achieved ICC scores of 0.943 and 0.967 among fellow and junior attending radiologists, respectively [11]. The proposed DL algorithm achieved an ICC score comparable to junior attending radiologists from the study when measuring the same set of 244 lesions. In a study by Tang et al., a convolutional neural network-based method for semi-automated RECISTS measurement was proposed and assessed using 

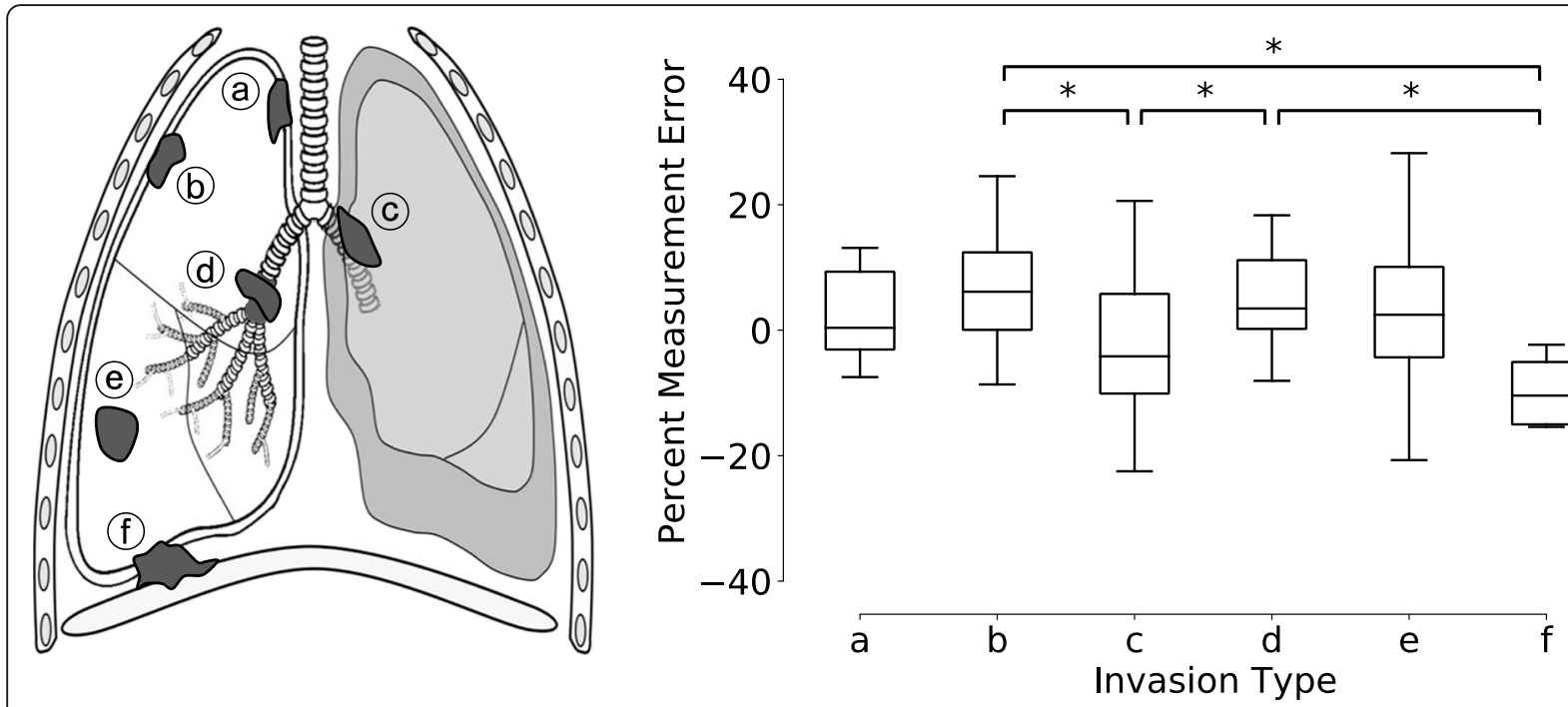

Fig. 6 DL algorithm's measurement error by tumor invasion type. The measurement error was calculated by using the following formula: $\frac{2 \bullet(\text { measurementol-measurement human })}{\left.\text { (measurement } t_{L}+\text { measurement thuman }\right)}$. The invasion types were classified as follows: a Parietal pleura/chest wall invasion. b Mediastinal pleural invasion. c Endobronchial invasion less than $2 \mathrm{~cm}$ distal to the carina. $\mathbf{d}$ Invasion associated with collapse (atelectasis). e Peripheral invasion surrounded by lung. $\mathbf{f}$ Diaphragm invasion. Box plots show the distribution of percent measurement errors stratified by invasion type. Bonferroni multiple comparison was performed, with statistical significance defined as $* P<0.05$

a mean difference between DL algorithm and radiologists in the unit of pixels (mean difference: 3.33 pixels; standard deviation: 4.93 pixels) [18]. Our model achieved a mean pixel difference and standard deviation of 2.85 and 2.51, which are 14 and $51 \%$ lower than the performance suggested by the study, respectively. However, the score using pixel difference may not be a reliable measure as the score is largely affected by the composition of the dataset; when a percent measurement difference between readers is fixed, having a larger number of larger lesions may inflate the performance score of DL algorithm. For example, given a pixel spacing of 0.1 and a lesion size of $5 \mathrm{~cm}$, the measurement difference of 5 pixels accounts only $10 \%$ measurement difference between two readers. On the contrary, given a pixel spacing of 0.1 and a lesion size of $2 \mathrm{~cm}$, the measurement difference of 5 pixels accounts $25 \%$ measurement difference between two readers. In this study, we primarily used Bland-Altman plotting based on percent measurement difference to address the issue.

Bland-Altman plotting suggested that the proposed algorithm generally yielded comparable measurements to a human radiologist with $240(98.4 \%)$ measurements realized within or around the upper and lower limits of agreement (LOA). Among the $4(1.6 \%)$ measurements outside the LOA, we observed that 2 deviating measurements (Fig. 5a and c) potentially subject to controversy among human observers, with some radiologists accepting the measurements and others rejecting them. The first lesion underestimated by the DL algorithm (Fig. 5a) was subject to controversy on whether its spiculations should be included in the measurement or not. This particular case highlighted the inherent difficulty in measuring lung lesion as well as lesion in other organs, as there is no clear consensus existing with regard to how the spiculations should be taken into account in lesion measurement. In the second underestimated lesion (Fig. 5b), the clinical reasoning behind the underestimation by the DL algorithm is unclear. The first overestimated measurement by DL algorithm (Fig. 5c) appeared to include the central density in its measurement while the density was not included in the measurement by the human radiologist. The controversy was associated with whether the central density should be seen as a blood vessel or part of the target lesion. In the second overestimated lesion (Fig. 5d), the algorithm failed to recognize two separate lesions sharing the same field of view and combined them into a single measurement.

As demonstrated, the Bland-Altman plotting indicated that the algorithm tends to marginally overestimate the size of tumor compared to the human radiologist. Further statistical test was performed to assess whether the algorithm's diagnostic behavior of under or overestimation is associated with the tumor types. It was observed that some tumor invasion types may induce the DL algorithm to over or underestimating the lesion size compared to the human radiologist. For example, the algorithm is likely to overestimate when measuring mediastinal pleural invasion (Fig. 6, Invasion Type b), compared to when measuring diaphragm invasion (Fig. 6, Invasion Type f) with the difference in its diagnostic behavior statistically significant. The lesion size measurement of some invasion types 
requires different clinical reasoning highly prone to interobserver variability. The systematic difference between the algorithm and human radiologist attributed by the lesionwise characteristics may or may not be due to interobserver variability between the trainer and tester radiologists.

This study had some limitations. First, this was a semiautomated method as the algorithm requires an arbitrary point within target lesion as an input. Given the recent advancements in automated detection of lung cancer, future studies may want to address the limitation by exploring a hybrid model that detects a lesion, identifies an arbitrary point inside the lesion, and performs measurement using the input point. Second, it has been well documented that methods utilizing neural networks are subject to a well-recognized challenge of their black-box nature $[37,38]$, which make it harder to fully explain the algorithm's measurement behavior. A further study utilizing techniques from interpretable machine learning may be explored to address the challenge $[39,40]$. Lastly, the presented study was designed with an emphasis on internal validity by comparing the algorithm with one radiologist who was ruled out during the training process. Future study designs may aim at generalizability by training and comparing the algorithm with multiple groups of radiologists using external validation data set.

\section{Conclusions}

This study proposed and validated a deep learning algorithm for semi-automated CT measurement of lung lesions. The DL algorithm yielded unidirectional measurements comparable to those of human radiologist and presented an excellent agreement. The DL algorithm was designed to work with any image with known patient-to-detector distance and the corresponding pixel spacing information, indicating a potential for its application in other anatomic measurements.

\section{Abbreviations}

ICC: Intraclass Correlation Coefficient; RECIST 1.1: Response Evaluation Criteria in Solid Tumors 1.1; DL: Deep Learning; DICOM: Digital Imaging and Communications in Medicine; LOA: Limits of agreement

\section{Supplementary Information}

The online version contains supplementary material available at https://doi. org/10.1186/s40644-021-00413-7.

\section{Additional file 1.}

\section{Acknowledgements}

The authors acknowledge and appreciate biostatistics support and mentorship of Professor Moonseong Heo, PhD of the Department of Public Health Sciences, Clemson University. We also acknowledge and appreciate the logistics and regulatory support of Karen Edwards, MS of the Department of Public Health Sciences, Clemson University.

\section{Authors' contributions}

MW designed the study, developed algorithms, and analyze data. MD, SL, and EL acted as Clinical Investigators and contributed substantially to study development, clinical data preparation, collection, and interpretation. RG served as co-PI and supervised preparation, conduct, and administration of the study. All authors developed, reviewed and approved the manuscript.

\section{Funding}

This work is based on the research supported by Health Science Center, Prisma Health, Greenville, South Carolina.

\section{Availability of data and materials}

The datasets utilized during the current study are available in The Cancer Imaging Archive, https://www.cancerimagingarchive.net/.

\section{Declarations}

\section{Ethics approval and consent to participate}

The study protocol was approved by the Institutional Review Board of Prisma Health, Greenville, South Carolina (Pro00077010). The study did not involve human subjects.

\section{Consent for publication}

Not applicable.

Competing interests

Not applicable.

\section{Author details}

'Department of Public Health Sciences, Clemson University, 501 Edwards Hall, Clemson, SC 29634, USA. ²Department of Radiology, Prisma Health System, 200 Patewood Drive, Greenville, SC 29615, USA.

Received: 18 September 2020 Accepted: 9 June 2021

Published online: 23 June 2021

\section{References}

1. Eisenhauer EA, Therasse P, Bogaerts J, Schwartz LH, Sargent D, Ford R, et al. New response evaluation criteria in solid tumours: revised RECIST guideline (version 1.1). Eur J Cancer. 2009:45(2):228-47.

2. Schwartz LH, Litière $S$, de Vries E, Ford R, Gwyther S, Mandrekar S, et al. RECl ST 1.1 - Update and clarification: From the RECIST committee. Eur J Cancer. 2016;62:132-7.

3. Schwartz LH, Seymour L, Litière S, Ford R, Gwyther S, Mandrekar S, et al. RECIST 1.1-Standardisation and disease-specific adaptations: Perspectives from the RECIST Working Group. Eur J Cancer. 2016;62:138-45.

4. Therasse $P$, Eisenhauer E, Verweij J. RECIST revisited: a review of validation studies on tumour assessment. Eur J Cancer. 2006;42(8):1031-9.

5. Woo M, Lowe SC, Devane AM, Gimbel RW. Intervention to Reduce Interobserver Variability in Computed Tomographic Measurement of Cancer Lesions Among Experienced Radiologists. Curr Probl Diagn Radiol. 2021; 50(3):321-7

6. Yoon SH, Kim KW, Goo JM, Kim DW, Hahn S. Observer variability in RECISTbased tumour burden measurements: a meta-analysis. Eur J Cancer. 2016;53: 5-15.

7. Sosna J, IS RECIST. Version 1.1 Reliable for Tumor Response Assessment in Metastatic Cancer? Radiology. 2019;290(2):357-8.

8. Jiang B, Zhou D, Sun Y, Wang J. Systematic analysis of measurement variability in lung cancer with multidetector computed tomography. Ann Thorac Med. 2017;12(2):95-100.

9. Singh S, Maxwell J, Baker JA, Nicholas JL, Lo JY. Computer-aided classification of breast masses: performance and interobserver variability of expert radiologists versus residents. Radiology. 2011;258(1):73-80.

10. Coy HJ, Douek ML, Ruchalski K, Kim HJ, Gutierrez A, Patel M, et al. Components of Radiologic Progressive Disease Defined by RECIST 1.1 in Patients with Metastatic Clear Cell Renal Cell Carcinoma. Radiology. 2019; 292(1):103-9.

11. McErlean A, Panicek DM, Zabor EC, Moskowitz CS, Bitar R, Motzer RJ, et al. Intra-and interobserver variability in CT measurements in oncology. Radiology. 2013;269(2):451-9. 
12. Woo M, Heo M, Devane AM, Lowe SC, Gimbel RW. Retrospective comparison of approaches to evaluating inter-observer variability in $\mathrm{CT}$ tumour measurements in an academic health centre. BMJ Open. 2020; 10(11):e040096.

13. Hirata K, Wong K-P, Kobayashi K, Manabe O, Tamaki N, Huang S-C. Metavol: a semi-automated software tool for whole-body tumor volume measurement in FDG PET-CT. J Nucl Med. 2014;55(supplement 1):1369.

14. Brown MS, Kim HJ, Lo P, Barnoy E, McNitt-Gray MF, Aberle DR, et al. Automated tumor size assessment: Consistency of computer measurements with an expert panel. J Clin Oncol. 2013;31(15_suppl):7566.

15. Meier R, Knecht U, Loosli T, Bauer S, Slotboom J, Wiest R, et al. Clinical evaluation of a fully-automatic segmentation method for longitudinal brain tumor volumetry. Sci Rep. 2016;6:23376.

16. Vorontsov E, Cerny M, Régnier P, Di Jorio L, Pal CJ, Lapointe R, et al. Deep learning for automated segmentation of liver lesions at $C T$ in patients with colorectal cancer liver metastases. Radiology. 2019;1(2):180014.

17. Chlebus G, Schenk A, Moltz JH, van Ginneken B, Hahn HK, Meine H. Automatic liver tumor segmentation in $\mathrm{CT}$ with fully convolutional neural networks and object-based postprocessing. Sci Rep. 2018;8(1):15497.

18. Tang Y, Harrison AP, Bagheri M, Xiao J, Summers RM, editors. Semiautomatic RECIST labeling on CT scans with cascaded convolutional neural networks. International Conference on Medical Image Computing and Computer-Assisted Intervention; 2018: Springer.

19. Clark K, Vendt B, Smith K, Freymann J, Kirby J, Koppel P, et al. The Cancer Imaging Archive (TCIA): maintaining and operating a public information repository. J Digit Imaging. 2013;26(6):1045-57.

20. Aerts H, Velazquez ER, Leijenaar RT, Parmar C, Grossmann P, Cavalho S, et al. Data from NSCLC-radiomics. Cancer Imaging Arch. 2016.

21. Consortium NCICPTA. Radiology data from the clinical proteomic tumor analysis consortium lung squamous cell carcinoma [CPTAC-LSCC] collection. Cancer Imaging Arch. 2018

22. Yang J, Sharp G, Veeraraghavan $\mathrm{H}$, van Elmpt W, Dekker A, Lustberg T, et al. Data from lung $C T$ segmentation challenge. Cancer Imaging Arch. 2017.

23. Goldgof D, Hall L, Hawkins S, Schabath M, Stringfield O, Garcia A, et al. Data From QIN_LUNG_CT. Cancer Imaging Arch. 2017

24. Albertina B, Watson M, Holback C, Jarosz R, Kirk S, Lee Y, et al. Radiology data from the cancer genome atlas lung adenocarcinoma [tcga-luad] collection. Cancer Imaging Arch. 2017.

25. Armato SG III, Hadjiiski L, Tourassi GD, Drukker K, Giger ML, Li F, et al. SPIEAAPM-NCI Lung Nodule Classification Challenge Dataset. Cancer Imaging Arch. 2016.

26. Grove $\mathrm{O}$, Berglund $\mathrm{AE}$, Schabath $\mathrm{MB}$, Aerts $\mathrm{H}$, Dekker $\mathrm{A}$, Wang $\mathrm{H}$, et al. Data from: Quantitative computed tomographic descriptors associate tumour shape complexity and intratumor heterogeneity with prognosis in lung adenocarcinoma. Cancer Imaging Arch. 2015.

27. Zhao B, Schwartz LH, Kris MG. Data from RIDER_Lung CT. Cancer Imaging Arch. 2015.

28. Willemink MJ, Koszek WA, Hardell C, Wu J, Fleischmann D, Harvey H, et al. Preparing medical imaging data for machine learning. Radiology. 2020; 295(1):4-15

29. Tabibu S, Vinod PK, Jawahar CV. Pan-Renal Cell Carcinoma classification and survival prediction from histopathology images using deep learning. Sci Rep. 2019;9(1):10509.

30. Chang GH, Felson DT, Qiu S, Guermazi A, Capellini TD, Kolachalama VB. Assessment of knee pain from MR imaging using a convolutional Siamese network. Eur Radiol. 2020;30(6):3538-48.

31. Keys R. Cubic convolution interpolation for digital image processing. IEEE Trans Acoust Speech Signal Process. 1981;29(6):1153-60.

32. Amruta A, Gole A, Karunakar Y, editors. A systematic algorithm for 3-D reconstruction of MRI based brain tumors using morphological operators and bicubic interpolation. 2010 2nd International Conference on Computer Technology and Development; 2010: IEEE.

33. Shorten C, Khoshgoftaar TM. A survey on image data augmentation for deep learning. J Big Data. 2019;6(1):60.

34. Shrout PE, Fleiss JL. Intraclass correlations: uses in assessing rater reliability. Psychol Bull. 1979;86(2):420

35. Sandstedt M, Henriksson L, Janzon M, Nyberg G, Engvall J, De Geer J, et al Evaluation of an Al-based, automatic coronary artery calcium scoring software. Eur Radiol. 2020;30(3):1671-8.
36. Choi Y, Lee IJ, Lee CY, Cho JH, Choi WH, Yoon HI, et al. Multi-institutional analysis of T3 subtypes and adjuvant radiotherapy effects in resected T3N0 non-small cell lung cancer patients. Radiat Oncol J. 2015;33(2):75-82.

37. Chaunzwa TL, Hosny A, Xu Y, Shafer A, Diao N, Lanuti M, et al. Deep learning classification of lung cancer histology using CT images. Sci Rep. 2021;11(1):5471.

38. Hosny A, Parmar C, Coroller TP, Grossmann P, Zeleznik R, Kumar A, et al. Deep learning for lung cancer prognostication: A retrospective multi-cohort radiomics study. PLoS Med. 2018;15(11):e1002711.

39. Wang CJ, Hamm CA, Savic LJ, Ferrante M, Schobert I, Schlachter T, et al. Deep learning for liver tumor diagnosis part II: convolutional neural network interpretation using radiologic imaging features. Eur Radiol. 2019;29(7): 3348-57.

40. Guidotti R, Monreale A, Ruggieri S, Turini F, Giannotti F, Pedreschi D. A Survey of Methods for Explaining Black Box Models. ACM Comput Surv. 2018;51(5):Article 93.

\section{Publisher's Note}

Springer Nature remains neutral with regard to jurisdictional claims in published maps and institutional affiliations.
Ready to submit your research? Choose BMC and benefit from:

- fast, convenient online submission

- thorough peer review by experienced researchers in your field

- rapid publication on acceptance

- support for research data, including large and complex data types

- gold Open Access which fosters wider collaboration and increased citations

- maximum visibility for your research: over $100 \mathrm{M}$ website views per year

At $\mathrm{BMC}$, research is always in progress.

Learn more biomedcentral.com/submissions 